\title{
Serum and urinary calcium level in Latvian patients with sarcoidosis
}

\author{
leva Ruža ${ }^{1}$, Zane Lucāne ${ }^{2}$ \\ ${ }^{1}$ Department of Endocrinology, Riga Eastern Clinical University Hospital, Latvia \\ ${ }^{2}$ Department of Continuing Education, Riga Eastern Clinical University Hospital, Latvia
}

\begin{abstract}
Objectives: Sarcoidosis is a multisystem granulomatous disease of unknown etiology that in $90 \%$ of cases affects the lungs. Calcium metabolism testing can be useful in diagnostics. The aim of the study was to assess the correlation between calcium metabolism and sarcoidosis form of manifestation/demographic indicators.

Material and methods: In a retrospective study medical records of all patients ( $n=699)$ who had been hospitalized with suspected sarcoidosis in a specialized clinic of Riga Eastern Clinical University Hospital during the period from January $1^{\text {st }}, 2013$ until December 31 $31^{\text {st }}, 2014$ were analyzed. Further analysis included only patients with histologically and/or clinically confirmed sarcoidosis $(n=281)$. Results: Patients' average age at the time of diagnosis was $39 \pm 13$ years. Elevated serum calcium was observed in $9.9 \%$ of cases. A statistically significant correlation was found between serum calcium and age $(p<0.01)$. There was an association between serum calcium and gender $(p<0.05)$ - levels were higher in men $(2.43 \mathrm{mmol} / \mathrm{l})$ than in women $(2.40 \mathrm{mmol} / \mathrm{l})$. Elevated calcium in 24-hour urine was observed in $22.7 \%$ of patients. The mean value was $232.3 \mathrm{mg} / 24 \mathrm{~h}$, levels were higher in men $(258.7 \mathrm{mg} / 24 \mathrm{~h})$ than in women $(202.3 \mathrm{mg} / 24 \mathrm{~h})$, and the association with gender was statistically significant $(p<0.01)$.

Conclusions: We can conclude that in Latvia sarcoidosis affects mostly young and middle-aged people. Both serum calcium and calcium in 24-hour urine are important parameters for sarcoidosis diagnostics. Hypercalcemia was found in $9.9 \%$ of patients, hypercalciuria in $22.7 \%$ of patients, and both were statistically significantly higher in men, regardless of age.
\end{abstract}

Key words: sarcoidosis, hypercalcemia, serum calcium level.

\section{Introduction}

Sarcoidosis is a granulomatous inflammatory disease with clinical manifestations varying from spontaneously regressive localized forms to chronic disease with multiorgan involvement [1]. Lung sarcoidosis is the most common form of the disease, but any organ system can be involved. The most common manifestations are a persistent cough, erythema nodosum, eye involvement or peripheral lymph node form, fatigue and incidental finding on the chest X-ray [2].

The etiology of sarcoidosis remains unknown, but dysregulated immune reactions are at the core of the pathologic mechanism of the disease. T-cell infiltration is present, and $\mathrm{T} 1$ helper lymphocyte release increased amounts of cytokines in the case of sarcoidosis [3]. The antigen that initiates and drives the immune response in sarcoidosis has not been identified [4]. The main histological feature of sarcoidosis is the formation of non-caseous granulomas, which can persist, spontaneously regress or lead to fibrosis [5].

A well-known complication of sarcoidosis is dysregulated vitamin D and calcium metabolism. There is increased synthesis of calcitriol in the sarcoid granulomas, and this can lead to hypercalcemia and hypercalciuria. In

Address for correspondence:

leva Ruža, Department of Endocrinology, Riga Eastern Clinical University Hospital, 2 Hipokrata St., LV 1038, Riga, Latvia,

e-mail: dr.ieva.ruza@gmail.com

Submitted: 18.09.2018; Accepted: 3.12.2018 
the case of sarcoidosis, INF- $\gamma$, synthesized by T1 helper lymphocytes, stimulates $1 \alpha$-hydroxylase in alveolar macrophages, which leads to increased production of calcitriol [6]. In renal tubular cells, high levels of calcitriol reduce the activity of $1 \alpha$-hydroxylase and increase the activity of 24-hydroxylase. 24,25-dihydroxycholecalciferol is a metabolically inactive form of vitamin D. In the case of sarcoidosis, activity of $1 \alpha$-hydroxylase is not inhibited by increased levels of calcitriol. At the same time, macrophages do not express normally functioning 24-hydroxylase, which means that these cells lack calcitriol level reducing activity [7]. Calcitriol stimulates absorption of calcium in the intestine (hyperabsorptive hypercalcemia) and promotes hypercalcemia with a suppressed level of parathyroid hormone. It also stimulates osteoclasts to induce bone resorption, and osteoblasts to induce the formation of bone [7, 8]. Calcitriol attaches to calcitriol receptors, a member of the nuclear receptor family of transcription factors. Calcitriol has multiple effects on immunity regulation and initiation [6].

The approximate prevalence of hypercalcemia in sarcoidosis patients has been estimated to be 2 to $27 \%$ [9]. Severe hypercalcemia is rare in sarcoidosis [10]. However, in rare cases, severe hypercalcemia, defined as a calcium level of $3.5 \mathrm{mmol} / \mathrm{l}$, can have clinically serious consequences [7]. According to some authors, increased intake of exogenous vitamin D or increased exposure to sunlight can intensify the problem [10]. Another mechanism, apart from the dysregulated metabolism of vitamin D for hypercalcemia in sarcoidosis patients, is related to synthesis of the parathyroid hormone related hormone in the granulomas [1].

Hypercalciuria is the most common defect in the calcium metabolism in patients with sarcoidosis and the most common renal manifestation. Prevalence of hypercalciuria varies from 40 to $62 \%$ [7]. Possible consequences of hypercalciuria include kidney stone disease, deposits of calcium in renal parenchyma, renal failure or tubular pathologies, including nephrogenic diabetes insipidus [11].

According to some authors, sarcoidosis patients should limit the daily intake of calcium and vitamin D due to abnormal vitamin D and calcium metabolism [12].

The aim of the study was to assess the correlation between calcium metabolism and sarcoidosis form of manifestation and demographic indicators.

\section{Material and methods}

Almost all patients with primarily diagnosed sarcoidosis in Latvia - a Northern European country [13] - have been investigated in the "Centre of Tuberculosis and Lung Diseases" of Riga Eastern Clinical University
Hospital. In a retrospective study medical records of all patients ( $n=699$ ) who had been hospitalized in the Riga Eastern University Hospital's Centre of Tuberculosis and Lung Diseases due to suspected sarcoidosis during the period from the 1st of January 2013 until the $31^{\text {st }}$ of December 2014 were analyzed. Data were collected using the computerized hospital information system and patients' medical records in November 2015.

Inclusion criteria were the following: 1) a patient older than 18 years of age, and 2) a patient with histologically and/or clinically approved (e.g. acute manifestation) diagnosis of sarcoidosis. Among all patients, 281 patients met the inclusion criteria. Data of calcium levels were analyzed in all 281 patients, comprising $50.5 \%(n=142)$ men and $49.5 \%(n=139)$ women. Calcium concentration in serum and urine was determined using commercially available assay kits that use a colorimetric method. Only total calcium testing results at the time of diagnosis from the medical records were obtained; no ionized values were available.

Hypercalcemia was defined as a calcium level of $2.55 \mathrm{mmol} / \mathrm{l}$ or higher. The reference range for calcium in 24-hour urine was 25 to $300 \mathrm{mg}$ in a 24-hour urine specimen ( 0.625 to $7.5 \mathrm{mmol} / \mathrm{d})$.

This study focuses mainly on the descriptive statistics. For independent samples, Student's t-test was performed in order to compare continuous data. Spearman's rank correlation coefficient was used to detect a significant correlation. All tests were two-tailed and hypothesis tests were deemed significant for $p<0.05$. All data were analyzed using Microsoft Office Excel and IBM SPSS Statistics for Windows version 22.0.

All procedures performed in studies involving human participants were in accordance with the ethical standards of the institutional research committee and with the 1964 Helsinki Declaration and its later amendments or comparable ethical standards. The study protocol was approved by the institutional review board of the Ethics Committee of Riga Stradins University (IRB no. $8 / 24.09 .2015)$. For this type of study formal consent is not required.

\section{Results}

A histologically verified diagnosis was made in 208 patients (109 men and 99 women). The age of patients ranged from 20 to 79 years. The patients' mean age at the time of diagnosis was $39.2 \pm 12.7$ years. Acute manifestation was found in $26 \%(n=73)$ of patients. Calcium in serum was tested in $79 \%(n=222)$ of patients $-76.8 \%$ of men $(n=109)$ and $81.3 \%$ of women $(n=113)$. The mean level of calcium in serum was $2.41 \pm 0.12 \mathrm{mmol} / \mathrm{l}$, and it ranged from 2.17 to $2.95 \mathrm{mmol} / \mathrm{l}$. Hypercalcemia, 
defined as an elevated level of serum calcium above 2.55 $\mathrm{mmol} / \mathrm{l}$, was observed in only $9.9 \%$ of cases $(n=22)$. None of the included patients had severe hypercalcemia, defined as a serum calcium level above $3.5 \mathrm{mmol} / \mathrm{l}$. A statistically significant correlation was found between serum calcium and patients' age $(p<0.01)$-younger patients were more likely to develop hypercalcemia (Fig. 1).

There was an association between serum calcium and gender $(p<0.05)$ - levels of calcium were higher in men (the mean value $2.43 \pm 0.10 \mathrm{mmol} / \mathrm{l}$ ) than in women (mean value $2.40 \pm 0.12 \mathrm{mmol} / \mathrm{l}$ ) (Fig. 2).

Calcium in 24-hour urine was tested in 69\% $(n=194)$ of patients $-72.5 \%$ of men $(n=103)$ and $65.5 \%$ of women $(n=91)$. The mean level of calcium in 24-hour urine was $232.3 \pm 136.5 \mathrm{mg} / 24 \mathrm{~h}(5.8 \mathrm{mmol} / \mathrm{d})$, and it ranged from 21.6 to $721.8 \mathrm{mg} / 24 \mathrm{~h}(0.5$ to $18 \mathrm{mmol} / \mathrm{d})$. Hypercalciuria, defined as urinary calcium excretion above $300 \mathrm{mg} / 24 \mathrm{~h}$ (7.5 mmol/d), was detected in $22.7 \%$ of patients $(n=44)$. Calcium in 24 -hour urine was significantly higher $(p<0.01)$ in men (mean value $258.7 \pm 144.3$ $\mathrm{mg} / 24 \mathrm{~h}(6.5 \mathrm{mmol} / \mathrm{d}))$ than in women (mean value $202.3 \pm 120.9 \mathrm{mg} / 24 \mathrm{~h}(5 \mathrm{mmol} / \mathrm{d})$ ) (Fig. 3).

No statistically significant correlation was found between hypercalciuria and age $(p=0.484)$, or serum and 24-hour urine calcium levels ( $p=0.203$ ), and no relation was found between acute or chronic first disease manifestation and levels of calcium in serum or in 24-hour urine $(p>0.5)$. Levels of parathyroid hormone and vitamin D (25-hydroxycholecalciferol and calcitriol) were tested in only a few patients $(n=5, n=4$ and $n=2$, respectively), not allowing any significant conclusions to be made. Primary hyperparathyroidism was neither confirmed nor excluded in those patients who developed hypercalcemia.

\section{Discussion}

In this study, we examined the prevalence of hypercalcemia and hypercalciuria in a sample of 281 patients newly diagnosed with sarcoidosis, and its relation to demographic indicators. We discovered that hypercalcemia occurs in $9.9 \%$ of newly diagnosed cases, which is consistent with previous studies of the prevalence of hypercalcemia in sarcoidosis patients, where it has been reported to be 2 to $27 \%$ [9].

In earlier studies, the prevalence of hypercalciuria was reported to be 40 to $67 \%$ [7], whereas in our study it was $22.7 \%$. No earlier data from Latvia have ever been published. A pilot project's results were presented as a poster in 2015 showing the prevalence of hypercalcemia and hypercalciuria at 8 and $23 \%$, respectively [14].

We also detected that men with sarcoidosis are more likely to have higher levels of serum calcium in comparison to women, which is consistent with the

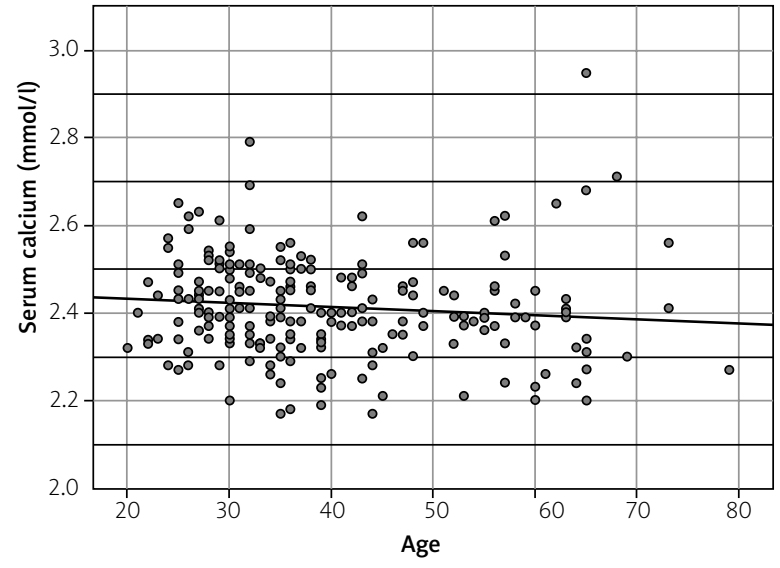

Fig. 1. Correlation between serum calcium (mmol/l) and patients' age.

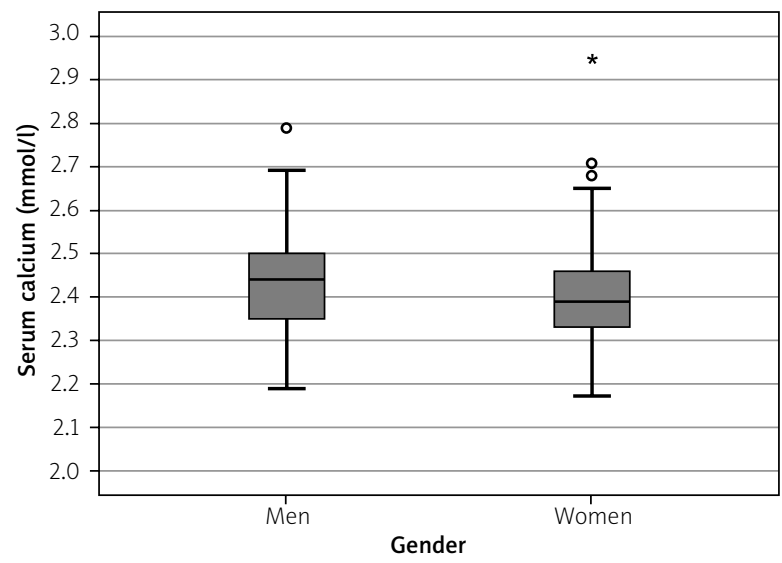

Fig. 2. Mean levels of serum calcium in men and women $(\mathrm{mmol} / \mathrm{l})$.

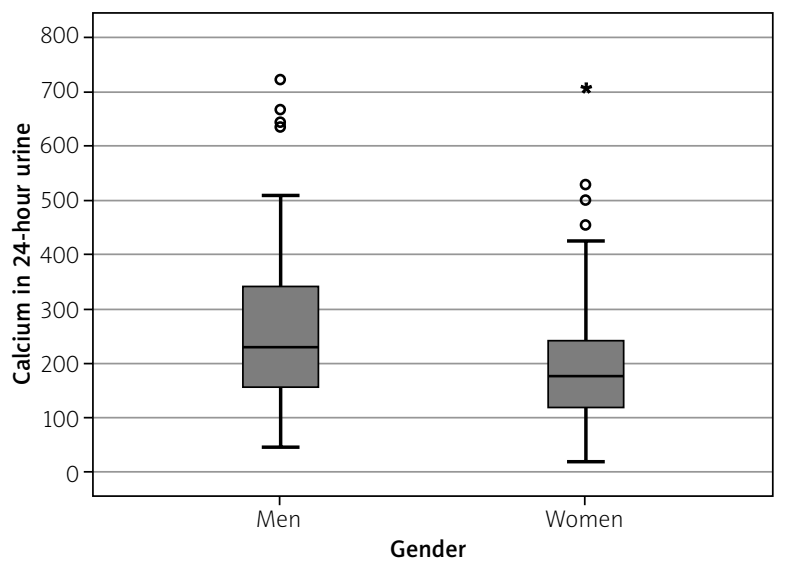

Fig. 3. Mean calcium in 24-hour urine specimen in men and women (mg/24 h). 
data reported from Estonia, another Northern European country [13], in 2016, where female patients were less likely to develop hypercalcemia in comparison to men, with an OR of 0.38 [15]. We did not find any significant relationship between levels of calcium (in serum or in urine) and manifestation form of the disease (acute or chronic).

Nephrolithiasis is a common renal manifestation of sarcoidosis. In a recent study, calcium in urine was higher in patients with nephrolithiasis, but serum 25-hydroxycholecalciferol and calcitriol did not correlate with urine calcium levels [16]. Coexistence of active sarcoidosis and primary hyperparathyroidism can be a diagnostic challenge, as hypercalcemia can coincide with normal or decreased parathyroid hormone values [17].

We found that, due to financial restrictions in the hospital and not the primary role in sarcoidosis diagnostics, vitamin D (25-hydroxycholecalciferol and calcitriol) and parathyroid hormone were detected in a very smal number of patients, postponing assessment to the outpatient period. Yet in recent years the question has remained open about prophylactic calcium and vitamin D supplementation in patients with coexisting sarcoidosis and any other health condition (e.g. glucocorticoid-induced or postmenopausal osteoporosis) requiring such intervention, especially in countries with reported higher vitamin D insufficiency in the general population [18-20].

Glucocorticoids are considered to be the first line medication for sarcoidosis requiring treatment, and osteoporosis is a well-known complication of prolonged use of glucocorticoids [21]. Vitamin D supplementation, which is considered to be a prophylactic measure to prevent osteoporosis, can influence calcium metabolism [22]. According to some authors, increased intake of exogenous vitamin D or increased exposure to sunlight can intensify the problem of hypercalcemia [10].

The question of supplementation is especially important in Latvia, a Northern European country [13] It has been reported that the prevalence of vitamin $D$ insufficiency in the general population is high in Latvia (latitude $56^{\circ}$ ). In a study conducted in 2007 the mean level of 25-hydroxycholecalciferol was $32.8 \mathrm{ng} / \mathrm{ml}$, with significantly lower levels detected in winter when compared with those in summer $(28.2 \mathrm{ng} / \mathrm{ml}$ vs. $37.5 \mathrm{ng} /$ $\mathrm{ml}$, respectively; $p<0.05$ ) [23]. In another study in 2015 the mean serum level of 25-hydroxycholecalciferol was $17.8 \mathrm{ng} / \mathrm{ml}$ in winter, $21.1 \mathrm{ng} / \mathrm{ml}$ in summer [24], and vitamin D supplementation was recommended for this population $[23,24]$. A retrospective study conducted in 2014, which included 301 patients with sarcoidosis, revealed that calcium and vitamin D supplementation is safe in patients with sarcoidosis, and is not related to an increased risk of hypercalcemia, and can be applied for patients receiving glucocorticoids, and in patients with risk of osteoporosis [18].

Another randomized, placebo-controlled study in the year 2013 revealed that in sarcoidosis patients with the level of 25 -hydroxycholecalciferol under $50 \mathrm{nmol} / \mathrm{l}$, additional vitamin D supplementation does not significantly affect levels of serum calcium or daily calcium excretion in urine [19]. However, a decreased level of serum 25-hydroxycholecalciferol in patients with sarcoidosis does not represent vitamin D deficiency, but the level is decreased due to increased vitamin D conversion in the active calcitriol form [25]. The serum concentration ratio between 25-hydroxycholecalciferol and calcitriol is increased in patients with sarcoidosis in comparison with the general population [12]. Also, a retrospective cohort study of 196 patients showed that those patients with sarcoidosis who received vitamin $D$ supplementation developed hypercalcemia more often than patients who did not receive such supplementation (43.3\% vs. 18.3\%) [9].

Another hypothesis states that vitamin $D$ deficiency could be the cause of an increased prevalence of sarcoidosis in Northern Europe (decreased exposure to the sunlight) and in the African American population (pigmentation filters UVB rays). A decreased level of vitamin D could exist before the manifestation of the disease, and this could have a role in the immunopathogenesis of sarcoidosis [26]. A decreased level of 25-hydroxycholecalciferol is a well-known risk factor for higher activity of several inflammatory diseases, including Crohn's disease and multiple sclerosis [18].

Saidenberg-Kermanac'h et al. [1] in 2014 reported that low level of 25-hydroxycholecalciferol is negatively correlated with the activity of sarcoidosis. However, a recent study that compared vitamin D status and calcium metabolism in two ethnic groups of patients (Caucasian patients in Italy and African American patients in the USA) concluded that the risk of vitamin D deficiency is not increased in sarcoidosis patients in comparison with the general population, nor there was found a difference in vitamin $\mathrm{D}$ and calcium metabolism between the two ethnic populations [16].

Therefore, the question about prophylactic calcium and vitamin D supplementation in patients with a risk of glucocorticoid-induced osteoporosis (or any other cause of osteoporosis, such as postmenopausal) remains unclear. It is important to assess the risk individually and to apply appropriate prophylactic measures. However, when vitamin D and calcium supplementation is used in patients with sarcoidosis, control of calcium levels should always be done [27], since this study also shows that hypercalcemia and hypercalciuria are common in the case of sarcoidosis. The necessity of vitamin D supplementation should be carefully evaluated in patients 
with sarcoidosis. Serum levels of 25-hydroxycholecalciferol and calcitriol should be measured prior to initiation of vitamin D supplementation, and in the case of a normal level of 25-hydroxycholecalciferol therapy with vitamin D may be withheld [20].

A key strength of this study is the relatively large patient sample. Since this was a retrospective study, and data were extracted from medical records, it was not possible to obtain data about daily calcium intake. This could influence the results of urinary calcium excretion, because this variable also depends on the daily intake of calcium. Other possible causes of hypercalcemia, for example malignancy with bone metastasis or hyperparathyroidism, were not excluded in this study, which could interfere with the results of the calcium levels.

\section{Conclusions}

We can conclude that in Latvia - a Northern European country - sarcoidosis affects mostly young and middleaged people, which is consistent with general epidemiological data [28]. Both serum calcium and calcium in 24-hour urine are important parameters for sarcoidosis diagnostics. Hypercalcemia was found in $9.9 \%$ of patients, hypercalciuria in $22.7 \%$ of patients, and levels of calcium in serum and urine were statistically significantly higher in men, regardless of age.

The authors declare no conflict of interest.

\section{References}

1. Saidenberg-Kermanac'h N, Semerano L, Nunes $H$, et al. Bone fragility in sarcoidosis and relationships with calcium metabolism disorders: a cross sectional study on 142 patients. Arthritis Res Ther 2014; 16: R78.

2. Valeyre D, Prasse A, Nunes H, et al. Sarcoidosis. Lancet 2014; 383: 1155-1167.

3. Iannuzzi MC, Fontana JR. Sarcoidosis: clinical presentation, immunopathogenesis, and therapeutics. JAMA 2011; 305: 391-399.

4. Moller DR, Rybicki BA, Hamzeh NY, et al. Genetic, immunologic, and environmental basis of sarcoidosis. Ann Am Thorac Soc 2017; 14: S429-S436.

5. lannuzzi MC, Rybicki BA, Teirstein AS. Sarcoidosis. N Engl J Med 2007; 357: 2153-2165.

6. Kavathia D, Buckley JD, Rao D, et al. Elevated 1, 25-dihydroxyvitamin $D$ levels are associated with protracted treatment in sarcoidosis. Respir Med 2010; 104: 564-570.

7. Birnie D, Ha AC, Gula L, et al. Cardiac sarcoidosis. Clin Chest Med 2015; 36: 657-668.

8. Hewison, M. Vitamin D and the intracrinology of innate immunity. Mol Cell Endocrinol 2010; 321: 103-111.

9. Sodhi A, Aldrich T. Vitamin D supplementation: not so simple in sarcoidosis. Am J Med Sci 2016; 352: 252-257.

10. Rizzato, G. Clinical impact of bone and calcium metabolism changes in sarcoidosis. Thorax 1998; 53: 425-429.
11. Holmes J, Lazarus A. Sarcoidosis: extrathoracic manifestations. Dis Mon 2009; 55: 675-692.

12. Baughman RP, Lower EE. Goldilocks, vitamin D and sarcoidosis. Arthritis Res Ther 2014; 16: 111.

13. United Nations, Statistics Division. Standard Country or Area Codes for Statistical Use (Rev. 4), Series M: Miscellaneous Statistical Papers, No. 49, New York: United Nations, ST/ESA/STAT/SER.M/49/ Rev.4; 1999 (access: 9.09.2018). Available from: https://unstats. un.org/unsd/methodology/m49/.

14. Tarcinska R, Baklasova A, Ruza I, et al. Calcium metabolism in Latvian patients with newly diagnosed sarcoidosis [poster session presentation]. 14 ${ }^{\text {th }}$ European Congress of Internal Medicine; 2015 Oct 14-16; Moscow, Russian Federation. Available from: https://www. efim2015.org/public/uploads/2015/EFIM/sessions/Posters $\% 20$ Pulmonology-09-09.pdf.

15. Lill H, Kliiman K, Altraja A. Factors signifying gender differences in clinical presentation of sarcoidosis among Estonian population. Clin Respir J 2016; 10: 282-290.

16. Capolongo G, Xu LH, Accardo M, et al. Vitamin-D status and mineral metabolism in two ethnic populations with sarcoidosis. J Investig Med 2016; 64: 1025-1034.

17. Lim V, Clarke BL Coexisting primary hyperparathyroidism and sarcoidosis cause increased angiotensin-converting enzyme and decreased parathyroid hormone and phosphate levels. J Clin Endocrinol Metab 2013; 98: 1939-1945.

18. Kamphuis LS, Bonte-Mineur F, van Laar JA, et al. Calcium and vitamin D in sarcoidosis: is supplementation safe? J Bone Miner Res 2014; 29: 2498-2503.

19. Bolland MJ, Wilsher ML, Grey A, et al. Randomised controlled trial of vitamin D supplementation in sarcoidosis. BMJ Open 2013; 3: e003562.

20. Baughman RP, Papanikolaou I. Current concepts regarding calcium metabolism and bone health in sarcoidosis. Curr Opin Pulm Med 2017; 23: 476-481.

21. Adler RA, Funkhouser HL, Petkov VI, et al. Glucocorticoid-induced osteoporosis in patients with sarcoidosis. Am J Med Sci 2003;325:1-6.

22. Kling JM, Clarke BL, Sandhu NP. Osteoporosis prevention, screening, and treatment: a review. J Womens Health 2014; 23: 563-572.

23. Lejnieks A, Slaidina A, Zvaigzne A, et al. Vitamin D status and its seasonal variations and association with parathyroid hormone concentration in healthy women in Riga. Medicina (Kaunas, Lithuania) 2013; 49: 329-334.

24. Mukane M, Rasa I, Mukans M. First epidemiological study about vitamin D status and lifestyle factors in healthy young adults from Latvia Y: DLAT study (part I). Osteoporosis Int 2015; 26: P359.

25. Sweiss NJ, Lower EE, Korsten P, et al. Bone health issues in sarcoidosis. Curr Rheumatol Rep 2011; 13: 265-272.

26. Bucova $M$, Suchankova $M$, Tibenska E, et al. TREM-2 receptor expression increases with $25(\mathrm{OH}) \mathrm{D}$ vitamin serum levels in patients with pulmonary sarcoidosis. Mediators Inflamm 2015; 2015: 1-7.

27. Statement on sarcoidosis. Joint Statement of the American Thoracic Society (ATS), the European Respiratory Society (ERS) and the World Association of Sarcoidosis and Other Granulomatous Disorders (WASOG) adopted by the ATS Board of Directors and by the ERS Executive Committee. Am J Respir Crit Care Med 1999; 160: 736-755.

28. Arkema EV, Cozier YC. Epidemiology of sarcoidosis: current findings and future directions. Ther Adv Chronic Dis 2018; 9: 227-240. 